\title{
Measuring high-density built environment for public health research: Uncertainty with respect to data, indicator design and spatial scale
}

\author{
Guibo Sun, ${ }^{1}$ Chris Webster, ${ }^{1}$ Michael Y. Ni, ${ }^{2}$ Xiaohu Zhang ${ }^{3}$ \\ ${ }^{1}$ Healthy High Density Cities Lab, Faculty of Architecture, University of Hong Kong, Hong Kong; \\ ${ }^{2}$ School of Public Health, Li Ka Shing Faculty of Medicine, University of Hong Kong, Hong Kong; \\ ${ }^{3}$ Singapore-MIT Alliance for Research and Technology, Singapore
}

\begin{abstract}
Uncertainty with respect to built environment (BE) data collection, measure conceptualization and spatial scales is evident in urban health research, but most findings are from relatively lowdensity contexts. We selected Hong Kong, an iconic high-density city, as the study area as limited research has been conducted on uncertainty in such areas. We used geocoded home addresses
\end{abstract}

Correspondence: Xiaohu Zhang, Singapore-MIT Alliance for Research and Technology, 1 Create Way, Singapore.

Tel.: +65.66012826 - Fax: +65.66842118 .

E-mail: xiaohu@smart.mit.edu

Key words: High-density; Built environment; Spatial scale; Uncertainty; GIS; Hong Kong.

Contributions: GS designed the study, conducted the data analysis and drafted the first manuscript. CW contributed to the study design and writing of the manuscript. MN contributed to the study design and writing of the manuscript. XZ contribute the GIS python programming and writing of the manuscript.

Conflict of interest: the authors declare no potential conflict of interest.

Funding: GS is supported by the HKU 35th round of the PDF/RAP Fellowship and National Natural Science Foundation of China (41561035) and MN is supported by the Health and Medical Research Fund, Food and Health Bureau, Government of Hong Kong Special Administrative Region (13143421). The Hong Kong Jockey Club Charities Trust funded the establishment of the FAMILY Cohort from 2007 to 2014

Ethical approval: FAMILY Cohort has obtained approval by the Institutional Review Board of the University of Hong Kong/Hospital Authority Hong Kong West Cluster.

Received for publication: 20 November 2017.

Revision received: 20 February 2018.

Accepted for publication: 21 February 2018.

CCopyright G. Sun et al., 2018

Licensee PAGEPress, Italy

Geospatial Health 2018; 13:653

doi:10.4081/gh.2018.653

This article is distributed under the terms of the Creative Commons Attribution Noncommercial License (CC BY-NC 4.0) which permits any noncommercial use, distribution, and reproduction in any medium, provided the original author(s) and source are credited.
( $n=5732$ ) from a large population-based cohort in Hong Kong to extract BE measures for the participants' place of residence based on an internationally recognized BE framework. Variability of the measures was mapped and Spearman's rank correlation calculated to assess how well the relationships among indicators are preserved across variables and spatial scales. We found extreme variations and uncertainties for the 180 measures collected using comprehensive data and advanced geographic information systems modelling techniques. We highlight the implications of methodological selection and spatial scales of the measures. The results suggest that more robust information regarding urban health research in high-density city would emerge if greater consideration were given to BE data, design methods and spatial scales of the BE measures.

\section{Introduction}

Public health researchers have started to pay attention to built environment $(\mathrm{BE})$ with regard to preventive approaches against the global pandemic of obesity and related health problems resulting from physical inactivity (Lee et al., 2012; Sallis et al., 2016). Previous studies of relationship between BE and public health have, however, predominantly been conducted in relatively lowdensity city contexts (Rydin et al., 2012; Barton and Grant, 2013). The is now evidence of uncertainty due to spatial scales and conceptualizing measures (Clark and Scott, 2014), an issue which becomes amplified in high-density cities such as Hong Kong, Tokyo, Mumbai and Shanghai (Low et al., 2016). These megacities are widely known for their geographic and morphological BE heterogeneity, but the uncertainty in relation to constructing and interpreting BE measures needs further examination.

According to Longley et al. (2015), uncertainty arises since most representations of the world are incomplete, erroneous, out of date, subject to generalization or aberrant. The absence of complete BE data of high-density cities often hinder inferring the relationship between $\mathrm{BE}$ and health outcomes. For example, a crossnational study reported difficulties in measuring accessibility of public transport because of incomplete bus records in Bogotá and Hong Kong (Adams et al., 2014). With efforts focused on the development of street audit instruments to collect objective BE data (Sun et al., 2017), current indicators collected by audit instruments are unlikely to reveal variability of locations (Cerin et al., 2013). Only single scales have been used in studies of Hong Kong, for example research at level of the block (an area bounded by roads and their intersections) for body constitution and sedentary behaviour (Low et al., 2016) or a residence-based buffer and a 15minutes walking radius for walking and physical activity (Cerin et al., 2013; Lu et al., 2016). 
Geographic information systems (GIS) are widely used to measure BE, but conceptualizing such indicators varies considerably across disciplines. For example, street design can be measured and presented as different indicators, i.e. block length, block size, intersection density, street density, connected intersection ratio or link node ratio (where node signifies the point where roads meet or cross) (Berrigan et al., 2010; Cerin et al., 2011; Sallis et al., 2016). However, there is a lack of clear and precise operational definitions of BE measures (Handy, 2005; Forsyth et al., 2006) together with limited information issuing from uncertainty analysis of such indicators in high-density cities. Kwan (2012) notes that important ambiguities arise in measuring geographical context due to the spatial and temporal uncertainty of where, when and how long individuals experience environmental influences. Spatially, the uncertainty relates to the discussion of geographic scales in measuring spatial phenomena, as the modifiable areal unit problem (MAUP) of arbitrarily defined boundaries (Clark and Scott, 2014; Longley et al., 2015). Lack of attention in consistency, validity and reliability of spatial scale of the measures could result in mischaracterization of environment exposure of subjects (Handy, 2005; Brownson et al., 2009).

Hong Kong is a typical high-density city, where little research has been conducted on uncertainty analysis of BE indicators and health associations. We aimed to investigate the reliability of a wide spectrum of measures in this city charactering high-density BE in urban health studies across size and morphology of spatial measurement units thereby deriving urban health indicators. The study is expected to provide insights to analyzing uncertainty in BE measures in public health studies with rigorous stratified location sampling from a territorial-wide cohort.

\section{Materials and Methods}

The 5-D (density, diversity, design, destination accessibility, and distance to transit) internationally recognized framework (Ewing and Cervero, 2010; Ewing et al., 2015) was used as design conception for constructing BE indicators based on a comprehensive dataset collected from governmental and private sectors. The 5-D approach includes density, measured as the variable of interest per unit of area; diversity, pertaining to the number of land use classes in that area; design, measured as street patterns and related characteristics; destination accessibility, indicating ease of access to trip attractions; and distance to transit, measured as the number of stations or stops per unit area.

Our measures concerned a representative sampling of residential addresses from the Hong Kong FAMILY Cohort which consists of a composite sample from several sources including a population representative random-core sample (Leung et al., 2015). The cohort covers almost all Hong Kong neighbourhoods (99.8\%) enabling detailed spatial epidemiological studies linking BE to health and well-being at the individual, household and neighbourhood levels. Cohort studies take a holistic view of health, investigating its different dimensions including socio-demographics, anthropometrics, lifestyle and behavioural factors, measures of social capital and biomaterials, looking for effective public health and preventive approaches to improving physical, mental and social well-being (Leung et al., 2015).

\section{Population and study area}

Hong Kong is one of the most densely populated places in the world. According to the latest available census released from the Census and Statistics Department, Government of Hong Kong Special Administrative Region (SAR) (https://www. censtatd.gov.hk/hkstat/sub/so20.jsp), the inhabitation at the end of 20017 was over seven million people in an area covering 1068 $\mathrm{km}^{2}$. The ubiquitous building design in Hong Kong is a podium serving as a base platform with 2 to 4 floors, with several high-rise residential towers built above the podium (Shelton et al., 2011). More than $75 \%$ t of the land comprises of non-BE areas, while most $\mathrm{BE}$ exists between the waterfront and the mountains. The dwelling density of Hong Kong urban area is over 1,250 units per hectare. The shortage of flat land together with high land values have prompted buildings to develop vertically (Shelton et al., 2011). Six spatial buffering and zonal techniques and scales were used for each BE measure in order to understand their performance in local contexts.

\section{Data sources}

Table 1 shows a summary of data types and sources collected from Hong Kong governmental and private sectors and stored in shapefile format, including building outlines and heights, street

Table 1. Comprehensive data of the built environment in Hong Kong.

\begin{tabular}{|c|c|c|}
\hline Built environment & Data specification & Data source \\
\hline Building data & $\begin{array}{l}\text { Digital topographic maps in iB1000 (Scale 1:1000): } \\
\text { http:/www.landsd.gov.hk/download.php?file=mapping/en/digital_ } \\
\text { map/common/feature/ibl_fgdb_mf.pdf }\end{array}$ & Lands Department of Hong Kong SAR \\
\hline Destination data & $\begin{array}{l}\text { Points of interest }(\mathrm{N}=329,644) \text { : } \\
\text { GeoCommunity database and an online map services. } \\
\text { http://www.landsd.gov.hk/download.php?file=mapping/en/digital_ } \\
\text { map/common/feature/geocom_mf.pdf }\end{array}$ & $\begin{array}{l}\text { Lands Department of Hong Kong SAR } \\
\text { Gaode.com }\end{array}$ \\
\hline Street network & $\begin{array}{l}\text { Intelligent road network package: i) road network data: } \\
\text { http://www.td.gov.hk/filemanager/en/content_4421/121130\% } \\
\text { 20package_rdnet_-mdb_vasp.pdf } \\
\text { ii) digitized traffic aids: } \\
\text { http://www.td.gov.hk/filemanager/en/content_4421/110527\%20package_ } \\
\text { dtad_-mdb.pdf }\end{array}$ & Transport Department of Hong Kong SAR \\
\hline Census tracts & $\begin{array}{l}\text { Tertiary planning unit and Street block/village cluster: } \\
\text { https://www.devb.gov.hk/filemanager/en/content_384/frv2E.pdf }\end{array}$ & Planning Department of Hong Kong SAR \\
\hline
\end{tabular}

SAR, Special Administrative Region. 
network, destinations from points of interest $(\mathrm{N}=329,644)$ and census tracts. The building density as floor area ratio, i.e. the ratio of a building's total floor area to the size of the piece of land upon which it is built, measured using the street-block census tract, is shown in Figure 1. Most land parcels in northern Hong Kong Island and Kowloon have a building density $>5$, which means that if the land of a block census tract is completely built, the number of floors is greater than 5 with the fifth quantile of building density being between 5 and 108 floors (Figure 1).

The residential addresses of FAMILY Cohort participants as of 2011 were geocoded. The detailed study design of the cohort has been described by Leung et al. (2015). The cohort comprised of 20,279 households and 46,001 participants. In this study, we used 5732 geocoded home addresses which excludes repeated addresses (different apartments) within the same building.

\section{Design of built environment measures}

Land use patterns refer to the spatial distribution of areas devoted to different purposes. Using the 5-D model, we measured three main dimensions of the high-density built environment: land use patterns, transport and urban design (Handy et al., 2002). The former was estimated, first by calculating the building density (i.e. total floor areas of podium and residential towers divided by the catchment area) and then destination accessibility, which were classified into eleven categories as displayed in Table 2. The destination accessibility measures were constructed using density and quantity where the density measure was derived from the quantity of accessible destinations divided by the catchment area.

We also constructed a measure of the mixture of destination and land-use pattern. The formula used to calculate this mixture is a variation of the entropy formula used by Frank and his colleagues (Frank et al., 2005).

$$
D M=-1\left(\sum_{i=1}^{n} d i * \ln (d i)\right) / \ln (n)
$$

where $D M$ is the mixture index, di the percentage of destination category $i$ within the study participant's catchment area and $n$ the number of destination categories.

We used street connectivity to measure urban design and trans- port, constructing several measures, including average and median lengths of a block (influenced by the design of the street network, density of the street intersections (a measure of network connectivity) and the link-node ratio (indicator of connectivity, which is equal to the number of links divided by the number of nodes within in a study area.

The shorter the block length, the more connected the community (Leslie et al., 2007), and the higher the intersection density or the link-node ratio, the more connected the network. The transport infrastructure accessibility is measured as the number of density and quantity of accessible transit stops, including public bus stops, metro entrances and ferries.

\section{Spatial scales}

Each BE measure was calculated at six spatial scale signified by: street-block group (SG), primary adjacency community (PAC), two circular buffers (CBs) and two network-based service area buffers (SAs) defined as shown in Figure 2 and Table 3. The SG

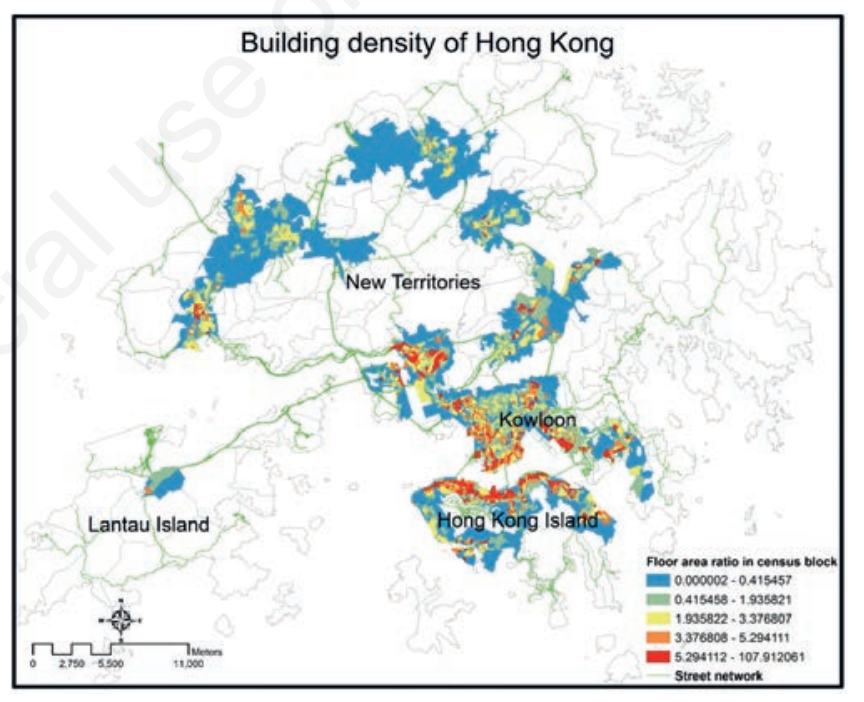

Figure 1. The density of Hong Kong built areas shown as streetblock census tract.

Table 2. Taxonomy of destinations.

\begin{tabular}{|c|c|}
\hline Destination category & Example \\
\hline $\begin{array}{l}\text { Small business } \\
\text { Groceries, supermarkets, } \\
\text { convenient stores }\end{array}$ & $\begin{array}{l}\text { Real estate intermediaries, small firms, information centres, lottery sales, travel agencies, express centres, etc. } \\
\text { Groceries, fruit and vegetable shops, farmer markets, supermarkets, convenience stores }\end{array}$ \\
\hline Entertainments & Sports \& leisure services, cinemas, scenic spots, hair and body spa centres, public green spaces, etc. \\
\hline Government and institutions & Governments, institutions, social organization centres, etc. \\
\hline Educational & Schools, libraries, scientific exhibition halls, training centres, convention centres, etc. \\
\hline Hotels & Hotels, hostels, etc. \\
\hline Retails & Shops, clothing and personal items shops, bookstores, gift shops, etc. \\
\hline Companies & Companies, industry factories, etc. \\
\hline Health care services & Hospitals, private clinics, community health care centres, etc. \\
\hline Financial services & Banks, insurance services, security company and centres, financial firms and service centres, etc. \\
\hline Restaurants & Restaurants, bars, coffees, dessert shops, etc. \\
\hline
\end{tabular}



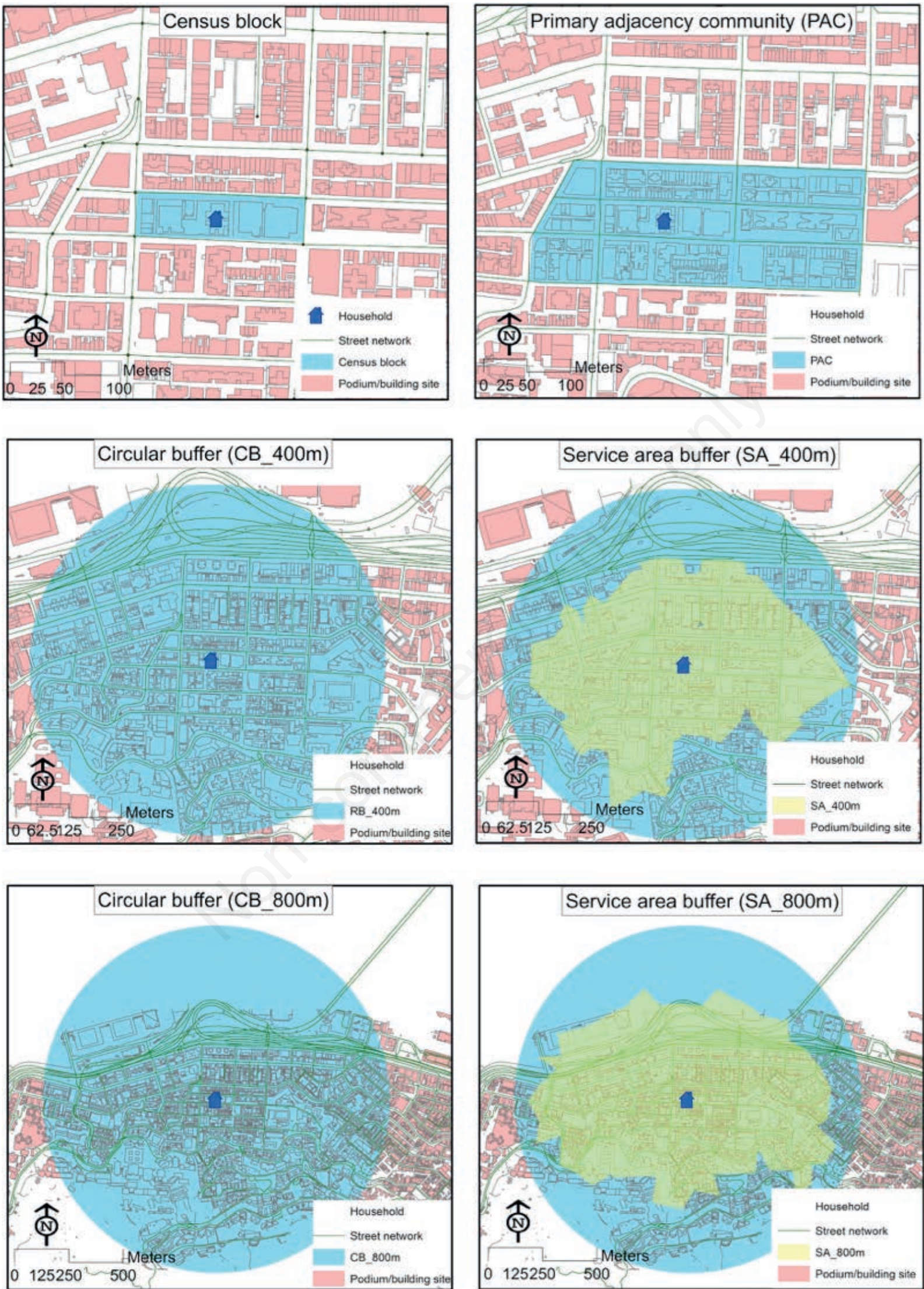

Figure 2. The six spatial scales. 
and PAC are zonal buffers consistent with urban design features and statistical census tracts. CBs and SAs are less related to morphological phenomena and more to behaviour using the crow-fly and network distances. In a literature review of studies using behaviour buffers to describe spatial contexts, 65\% used CBs while the rest used network-distances (Leal and Chaix, 2011).

\section{Constructing the built environment measures}

Density (1 indicator), diversity ( 1 indicator), design (4 indicators), destinations accessibility (22 indicators) and distance to transit ( 2 indicators) were calculated in the six spatial scales producing a total of $180 \mathrm{BE}$ indicators, extracted for study participants of the FAMILY Cohort using GIS (ArcGIS 10.31, ESRI, Redlands, CA, USA) and Python scripting with 64-bit desktop background geoprocessing (https://blogs.esri.com/esri/arcgis/ 2012/11/12/python-scripting-with-64-bit-processing/). Using GIS, we also mapped a destination mixture indicator and a density/quantity indictor of retail (as a destination) across spatial scales as examples to visualize the uncertainty in the design, choice and use of BE measures.

\section{Statistical analysis}

Descriptive statistics using Stata (MP 14, StataCorp LP, TX, USA) were applied to the BE measures and Spearman's rank correlation calculated to assess how well the relationships between two variables were persevered across spatial scales. We used various categories to evaluate rank preservation (Strominger et al., 2016) as follows: i) correlation $\geq 0.7$ indicates a well-preserved index; ii) correlation $\geq 0.5$ to 0.7 indicates a moderately preserved index; iii) correlation $\geq 0.3$ to 0.5 indicates a weakly preserved index; iv) correlation $<0.3$ indicates that the index is not preserved across scales.

\section{Results}

The results from the descriptive statistics are shown in Tables 4-6. Judged by the density measures the building density varied between the spatial scales. SA_400m showed the highest building density (4.0). It was different from the CB_400m (2.67) but similar

Table 3. Spatial scales investigated.

\section{Type of spatial scale Description}

Street-block group (SG)

The SG is the smallest census tract (average size $0.22 \mathrm{~km}^{2}$ ), which is demarcated by streets containing the 5,732 FAMILY Cohort unique building addresses (in podium/towers buildings). A SG contains one or a few closely adjacent buildings.

Primary adjacency community The PAC includes proximate communities including the home block and adjacent blocks that share boundaries defined by (PAC) a vertex or a line segment, usually a street (Strominger et al., 2016). The PAC represents the immediate environment of a cohort member, which is formed by the boundary of the closets streets.

Circular buffer (CB) The $\mathrm{CB}$ of the home address using a behaviourally determined impedance value (friction of distance) as radius. In this study, we used impedance values of $400\left(C B \_400 \mathrm{~m}\right)$ and $800 \mathrm{~m}\left(\mathrm{CB} \_800 \mathrm{~m}\right)$. These two radii correspond to short and long walking distances and have previously been applied in studies on the linkage of BE and activities such as walking, physical activity, well-being and chronic disease (Oliver et al., 2007).

Network-based service area buffer (SA)

The SA comprises areas that encompass all accessible streets tracing a given impedance value (e.g., 5 min or $400 \mathrm{~m}$ ) from a home address. SAs aim to provide a more accurate representation of spatial context than CBs. Also here, we used impedance values of 400 (SA_400m) and $800 \mathrm{~m}$ (SA_800m) as network radii to extract the BE measures referring to our study participants.

Table 4. Descriptive statistics of built environment characteristics at different scales.

\begin{tabular}{|c|c|c|c|c|c|c|c|c|c|c|c|c|}
\hline \multirow[t]{2}{*}{ Type of spatial scale } & \multicolumn{2}{|c|}{ SG } & \multicolumn{2}{|l|}{ PAC } & \multicolumn{2}{|c|}{ CB_400m } & \multicolumn{2}{|c|}{ CB_800m } & \multicolumn{2}{|c|}{ SA $400 \mathrm{~m}$} & \multicolumn{2}{|c|}{ SA $800 \mathrm{~m}$} \\
\hline & $\begin{array}{l}\text { Mean } \\
\text { (SD) }\end{array}$ & $\begin{array}{l}\text { Min } \\
\text { Max }\end{array}$ & $\begin{array}{l}\text { Mean } \\
\text { (SD) }\end{array}$ & $\begin{array}{l}\text { Min } \\
\text { Max }\end{array}$ & $\begin{array}{l}\text { Mean } \\
\text { (SD) }\end{array}$ & $\begin{array}{l}\text { Min } \\
\text { Max }\end{array}$ & $\begin{array}{l}\text { Mean } \\
\text { (SD) }\end{array}$ & $\begin{array}{l}\text { Min } \\
\text { Max }\end{array}$ & $\begin{array}{l}\text { Mean } \\
\text { (SD) }\end{array}$ & $\begin{array}{l}\text { Min } \\
\text { Max }\end{array}$ & $\begin{array}{l}\text { Mean } \\
\text { (SD) }\end{array}$ & $\begin{array}{l}\text { Min } \\
\text { Max }\end{array}$ \\
\hline Catchment area $\left(\mathrm{km}^{2}\right)$ & $\begin{array}{c}0.119 \\
(0.207)\end{array}$ & $\begin{array}{c}0.000616 \\
1.48\end{array}$ & $\begin{array}{c}1.07 \\
(1.63)\end{array}$ & $\begin{array}{l}0.0126 \\
11.600\end{array}$ & $\begin{array}{c}0.503 \\
(0)\end{array}$ & $\begin{array}{l}0.503 \\
0.503\end{array}$ & $\begin{array}{l}2.010 \\
(0)\end{array}$ & $\begin{array}{l}2.010 \\
2.01\end{array}$ & $\begin{array}{c}0.144 \\
(0.066)\end{array}$ & $\begin{array}{l}0.02 \\
0.424\end{array}$ & $\begin{array}{c}0.437 \\
(0.238)\end{array}$ & $\begin{array}{l}0.244 \\
(1.38)\end{array}$ \\
\hline $\begin{array}{l}\text { Density } \\
\text { Building density }\end{array}$ & $\begin{array}{c}3.77 \\
(2.14)\end{array}$ & $\begin{array}{c}0.00 \\
108.00\end{array}$ & $\begin{array}{l}2.08 \\
(1.67)\end{array}$ & $\begin{array}{c}0.00 \\
13.00\end{array}$ & $\begin{array}{c}2.67 \\
(1.33)\end{array}$ & $\begin{array}{c}0.00 \\
10.03\end{array}$ & $\begin{array}{l}1.85 \\
(0.97)\end{array}$ & $\begin{array}{l}0.05 \\
5.54\end{array}$ & $\begin{array}{c}4.00 \\
(1.91)\end{array}$ & $0.00-20.74$ & $\begin{array}{c}3.23 \\
(1.53)\end{array}$ & $\begin{array}{l}0.00 \\
11.09\end{array}$ \\
\hline $\begin{array}{l}\text { Diversity } \\
\text { Destination mixture }\end{array}$ & $\begin{array}{c}0.48 \\
(0.12)\end{array}$ & $\begin{array}{l}0.00 \\
1.00\end{array}$ & $\begin{array}{c}0.36 \\
(0.08)\end{array}$ & $\begin{array}{l}0.07 \\
0.69\end{array}$ & $\begin{array}{c}0.34 \\
(0.09)\end{array}$ & $\begin{array}{l}0.07 \\
1.00\end{array}$ & $\begin{array}{c}0.28 \\
(0.07)\end{array}$ & $\begin{array}{l}0.08 \\
0.58\end{array}$ & $\begin{array}{c}0.42 \\
(0.13)\end{array}$ & $0.00-1.00$ & $\begin{array}{c}0.36 \\
(0.12)\end{array}$ & $\begin{array}{l}0.00 \\
1.00\end{array}$ \\
\hline $\begin{array}{l}\text { Design } \\
\text { Average block length }\end{array}$ & $\begin{array}{l}118.00 \\
(76.30)\end{array}$ & $\begin{array}{c}15.00 \\
1130.00\end{array}$ & $\begin{array}{l}116.00 \\
(37.10)\end{array}$ & $\begin{array}{c}34.40 \\
680.00\end{array}$ & $\begin{array}{l}111.00 \\
(3670)\end{array}$ & $\begin{array}{c}57.10 \\
1420.00\end{array}$ & $\begin{array}{l}106.00 \\
(19.50)\end{array}$ & $\begin{array}{c}68.70 \\
451.00\end{array}$ & $\begin{array}{l}78.00 \\
(30.40)\end{array}$ & $29.50-1000.00$ & $\begin{array}{l}78.10 \\
(25.10)\end{array}$ & $\begin{array}{c}34.20 \\
734.00\end{array}$ \\
\hline Median block length & $\begin{array}{l}80.20 \\
(74.50)\end{array}$ & $\begin{array}{c}10.80 \\
1130.00\end{array}$ & $\begin{array}{l}64.10 \\
(16.10)\end{array}$ & $\begin{array}{c}19.70 \\
412.00\end{array}$ & $\begin{array}{c}65.30 \\
(19.70)\end{array}$ & $\begin{array}{l}21.30 \\
990.00\end{array}$ & $\begin{array}{l}60.70 \\
(9.34)\end{array}$ & $\begin{array}{c}36.80 \\
374.00\end{array}$ & $\begin{array}{l}52.30 \\
(25.40)\end{array}$ & $11.10-693.00$ & $\begin{array}{l}51.00 \\
(21.30)\end{array}$ & $\begin{array}{r}15.80 \\
792.00\end{array}$ \\
\hline Intersection density & $\begin{array}{c}0.00041 \\
(0.00042)\end{array}$ & $\begin{array}{c}0.00 \\
0.00508\end{array}$ & $\begin{array}{c}0.00034 \\
(0.00029)\end{array}$ & $\begin{array}{c}0.00 \\
0.00256\end{array}$ & $\begin{array}{c}0.00038 \\
(0.00022)\end{array}$ & $\begin{array}{c}0.00 \\
0.00132\end{array}$ & $\begin{array}{c}0.00030 \\
(0.00017)\end{array}$ & $\begin{array}{c}0.00 \\
0.00084\end{array}$ & $\begin{array}{c}0.00145 \\
(0.00087)\end{array}$ & $0.00-0.00525$ & $\begin{array}{c}0.00118 \\
(0.00063)\end{array}$ & $\begin{array}{c}0.00 \\
0.00472\end{array}$ \\
\hline Link-node ratio & $\begin{array}{l}0.85 \\
(0.36)\end{array}$ & $\begin{array}{l}0.53 \\
4.00\end{array}$ & $\begin{array}{l}0.62 \\
(0.09)\end{array}$ & $\begin{array}{l}0.52 \\
7.00\end{array}$ & $\begin{array}{c}0.57 \\
(0.03)\end{array}$ & $\begin{array}{l}0.51 \\
2.50\end{array}$ & $\begin{array}{l}0.53 \\
(0.01)\end{array}$ & $\begin{array}{l}0.51 \\
0.63\end{array}$ & $\begin{array}{c}0.59 \\
(0.07)\end{array}$ & $0.51-2.10$ & $\begin{array}{c}0.56 \\
(0.04)\end{array}$ & $\begin{array}{l}0.51 \\
1.12\end{array}$ \\
\hline
\end{tabular}

SG, Street-block group; PAC, primary adjacency community; CB, circular buffer; SA, service area buffer. 
Table 5. Destination accessibility. Descriptive statistics of destination accessibility with respect to density of destination $(i, i=1,2, \ldots, 11)$.

\begin{tabular}{|c|c|c|c|c|c|c|c|c|c|c|c|c|}
\hline \multirow{2}{*}{$\begin{array}{l}\text { Category } \\
\text { (See Table 2) }\end{array}$} & \multicolumn{2}{|c|}{ SG } & \multicolumn{2}{|c|}{ PAC } & \multicolumn{2}{|c|}{ CB_400m } & \multicolumn{2}{|c|}{ CB_800m } & \multicolumn{2}{|c|}{ SA_400m } & \multicolumn{2}{|c|}{ SA_800m } \\
\hline & $\begin{array}{l}\text { Mean } \\
\text { (SD) }\end{array}$ & $\begin{array}{l}\text { Min } \\
\text { Max }\end{array}$ & $\begin{array}{l}\text { Mean } \\
\text { (SD) }\end{array}$ & $\begin{array}{l}\text { Min } \\
\text { Max }\end{array}$ & $\begin{array}{l}\text { Mean } \\
\text { (SD) }\end{array}$ & $\begin{array}{l}\text { Min } \\
\text { Max }\end{array}$ & $\begin{array}{l}\text { Mean } \\
\text { (SD) }\end{array}$ & $\begin{array}{l}\text { Min } \\
\text { Max }\end{array}$ & $\begin{array}{l}\text { Mean } \\
\text { (SD) }\end{array}$ & $\begin{array}{l}\text { Min } \\
\text { Max }\end{array}$ & $\begin{array}{l}\text { Mean } \\
\text { (SD) }\end{array}$ & \\
\hline nall business & $\begin{array}{c}0.00021 \\
(0.00052)\end{array}$ & $\begin{array}{c}0.00 \\
0.00821\end{array}$ & $\begin{array}{c}0.00015 \\
(0.00033)\end{array}$ & $\begin{array}{c}0.00 \\
0.00550\end{array}$ & $\begin{array}{c}0.00014 \\
(0.00023)\end{array}$ & $\begin{array}{c}0.00 \\
0.00211\end{array}$ & $\begin{array}{c}0.00010 \\
(0.00014)\end{array}$ & $\begin{array}{c}0.00 \\
0.00090\end{array}$ & $\begin{array}{c}0.00017 \\
(0.00031)\end{array}$ & $\begin{array}{c}0.00 \\
0.00297\end{array}$ & $\begin{array}{c}0.00015 \\
(0.00025)\end{array}$ & $\begin{array}{c}0.00 \\
0.00259\end{array}$ \\
\hline $\begin{array}{l}\text { roceries, } \\
\text { nd supermarkets }\end{array}$ & $\begin{array}{c}0.00012 \\
(0.00025)\end{array}$ & $\begin{array}{c}0.00 \\
0.00517\end{array}$ & $(0.00011)$ & $\begin{array}{c}0.00 \\
0.00132\end{array}$ & $\begin{array}{c}0.00007 \\
(0.00006)\end{array}$ & $\begin{array}{c}0.00 \\
0.00036\end{array}$ & $\begin{array}{l}0.00005 \\
(0.00004)\end{array}$ & $\begin{array}{c}0.00 \\
0.00020\end{array}$ & $\begin{array}{c}0.00009 \\
(0.00010)\end{array}$ & $\begin{array}{c}0.00 \\
0.00089\end{array}$ & $\begin{array}{c}0.00008 \\
(0.00006)\end{array}$ & $\begin{array}{c}0.00 \\
0.00067\end{array}$ \\
\hline intertainments & $\begin{array}{c}0.00020 \\
(0.00047)\end{array}$ & $\begin{array}{c}0.00 \\
0.01480\end{array}$ & $\begin{array}{c}0.00014 \\
(0.00029)\end{array}$ & $\begin{array}{c}0.00 \\
0.00482\end{array}$ & $\begin{array}{c}0.00014 \\
(0.00020)\end{array}$ & $\begin{array}{c}0.00 \\
0.00160\end{array}$ & $\begin{array}{c}0.00010 \\
(0.00012)\end{array}$ & $\begin{array}{c}0.00 \\
0.00076\end{array}$ & $\begin{array}{c}0.00017 \\
(0.00028)\end{array}$ & $\begin{array}{c}0.00 \\
0.00369\end{array}$ & $\begin{array}{c}0.00015 \\
(0.00019)\end{array}$ & $\begin{array}{c}0.00 \\
0.00243\end{array}$ \\
\hline $\begin{array}{l}\text { ent } \\
\text { utions }\end{array}$ & $\begin{array}{c}0.00008 \\
(0.00021)\end{array}$ & $\begin{array}{c}0.00 \\
0.00969\end{array}$ & $\begin{array}{c}0.00006 \\
(0.00015)\end{array}$ & $\begin{array}{c}0.00 \\
0.00252\end{array}$ & $\begin{array}{c}0.00006 \\
(0.00010)\end{array}$ & $\begin{array}{c}0.00 \\
0.00071\end{array}$ & $\begin{array}{c}0.00004 \\
(0.00006)\end{array}$ & $\begin{array}{c}0.00 \\
0.00033\end{array}$ & $\begin{array}{c}0.00007 \\
(0.00013)\end{array}$ & $\begin{array}{c}0.00 \\
0.00180\end{array}$ & $\begin{array}{c}0.00006 \\
(0.00010)\end{array}$ & $\begin{array}{c}0.00 \\
0.00130\end{array}$ \\
\hline ducet & $\begin{array}{c}0.00012 \\
(0.00027)\end{array}$ & $\begin{array}{c}0.00 \\
0.00667\end{array}$ & $\begin{array}{c}0.00008 \\
(0.00015)\end{array}$ & $\begin{array}{c}0.00 \\
0.00302\end{array}$ & $\begin{array}{c}0.00009 \\
(0.00010)\end{array}$ & $\begin{array}{c}0.00 \\
0.00079\end{array}$ & $\begin{array}{c}0.00006 \\
(0.00006)\end{array}$ & $\begin{array}{c}0.00 \\
0.00039\end{array}$ & $\begin{array}{c}0.00011 \\
(0.00014)\end{array}$ & $\begin{array}{c}0.00 \\
0.00131\end{array}$ & $\begin{array}{c}0.00009 \\
(0.00010)\end{array}$ & $\begin{array}{c}0.00 \\
0.00119\end{array}$ \\
\hline Iotels & $\begin{array}{c}0.00004 \\
(0.00042)\end{array}$ & $\begin{array}{c}0.00 \\
0.01870\end{array}$ & $\begin{array}{c}0.00002 \\
(0.00015)\end{array}$ & $\begin{array}{c}0.00 \\
0.00527\end{array}$ & $\begin{array}{c}0.00002 \\
(0.00010)\end{array}$ & $\begin{array}{c}0.00 \\
0.00179\end{array}$ & $\begin{array}{c}0.00002 \\
(0.00007)\end{array}$ & $\begin{array}{c}0.00 \\
0.00062\end{array}$ & $\begin{array}{c}0.00003 \\
(0.00013)\end{array}$ & $\begin{array}{c}0.00 \\
0.00332\end{array}$ & $\begin{array}{c}0.00003 \\
(0.00009)\end{array}$ & $\begin{array}{c}0.00 \\
0.00132\end{array}$ \\
\hline Retails & $\begin{array}{c}0.00053 \\
(0.00123)\end{array}$ & $\begin{array}{c}0.00 \\
0.04210\end{array}$ & $\begin{array}{c}0.00041 \\
(0.00100)\end{array}$ & $\begin{array}{c}0.00 \\
0.01600\end{array}$ & $\begin{array}{c}0.00040 \\
(0.00068)\end{array}$ & $\begin{array}{c}0.00 \\
0.00563\end{array}$ & $\begin{array}{c}0.00029 \\
(0.00041)\end{array}$ & $\begin{array}{c}0.00 \\
0.00237\end{array}$ & $\begin{array}{c}0.00049 \\
(0.00093)\end{array}$ & $\begin{array}{c}0.00 \\
0.01250\end{array}$ & $\begin{array}{c}0.00042 \\
(0.00067)\end{array}$ & $\begin{array}{c}0.00 \\
0.00835\end{array}$ \\
\hline Companies & $\begin{array}{c}0.00062 \\
(0.00296)\end{array}$ & $\begin{array}{c}0.00 \\
0.05390\end{array}$ & $\begin{array}{c}0.00073 \\
(0.00210)\end{array}$ & $\begin{array}{c}0.00 \\
0.03240\end{array}$ & $\begin{array}{c}0.00070 \\
(0.00150)\end{array}$ & $\begin{array}{c}0.00 \\
0.01310\end{array}$ & $\begin{array}{c}0.00060 \\
(0.00096)\end{array}$ & $\begin{array}{c}0.00 \\
0.00555\end{array}$ & $\begin{array}{c}0.00076 \\
(0.00201)\end{array}$ & $\begin{array}{c}0.00 \\
0.02030\end{array}$ & $\begin{array}{c}0.00075 \\
(0.00161)\end{array}$ & $\begin{array}{c}0.00 \\
0.01590\end{array}$ \\
\hline Health care services & $\begin{array}{c}0.00018 \\
(0.00060)\end{array}$ & $\begin{array}{c}0.00 \\
0.03820\end{array}$ & $\begin{array}{c}0.00013 \\
(0.00038)\end{array}$ & $\begin{array}{c}0.00 \\
0.00953\end{array}$ & $\begin{array}{c}0.00013 \\
(0.00022)\end{array}$ & $\begin{array}{c}0.00 \\
0.00238\end{array}$ & $\begin{array}{c}0.00009 \\
(0.00014)\end{array}$ & $\begin{array}{c}0.00 \\
0.00084\end{array}$ & $\begin{array}{c}0.00016 \\
(0.00030)\end{array}$ & $\begin{array}{c}0.00 \\
0.00426\end{array}$ & $\begin{array}{c}0.00014 \\
(0.00022)\end{array}$ & $\begin{array}{c}0.00 \\
0.00247\end{array}$ \\
\hline Financial services & $\begin{array}{c}0.00008 \\
(0.00026)\end{array}$ & $\begin{array}{c}0.00 \\
0.00432\end{array}$ & $\begin{array}{c}0.00006 \\
(0.00016)\end{array}$ & $\begin{array}{c}0.00 \\
0.00359\end{array}$ & $\begin{array}{c}0.00006 \\
(0.00012)\end{array}$ & $\begin{array}{c}0.00 \\
0.00145\end{array}$ & $\begin{array}{c}0.00004 \\
(0.00007)\end{array}$ & $\begin{array}{c}0.00 \\
0.00057\end{array}$ & $\begin{array}{c}0.00008 \\
(0.00016)\end{array}$ & $\begin{array}{c}0.00 \\
0.00198\end{array}$ & $\begin{array}{c}0.00007 \\
(0.00013)\end{array}$ & $\begin{array}{c}0.00 \\
0.00189\end{array}$ \\
\hline Restaurants & $\begin{array}{c}0.00052 \\
(0.00102)\end{array}$ & $\begin{array}{c}0.00 \\
0.01540\end{array}$ & $\begin{array}{c}0.00033 \\
(0.00063)\end{array}$ & $\begin{array}{c}0.00 \\
0.00664\end{array}$ & $\begin{array}{c}0.00032 \\
(0.00042)\end{array}$ & $\begin{array}{c}0.00 \\
0.00318\end{array}$ & $\begin{array}{c}0.00022 \\
(0.00025)\end{array}$ & $\begin{array}{c}0.00 \\
0.00142\end{array}$ & $\begin{array}{c}0.00041 \\
(0.00058)\end{array}$ & $\begin{array}{c}0.00 \\
0.00601\end{array}$ & $\begin{array}{c}0.00035 \\
(0.00042)\end{array}$ & $\begin{array}{c}0.00 \\
0.00457\end{array}$ \\
\hline
\end{tabular}

SG, Street-block group; PAC, primary adjacency community; CB, circular buffer; SA, service area buffer.

Table 6. Descriptive statistics of destination accessibility with respect to quantity of destination $(i, i=1,2, \ldots, 11)$.

\begin{tabular}{|c|c|c|c|c|c|c|c|c|c|c|c|c|}
\hline \multirow{2}{*}{$\begin{array}{l}\text { Category } \\
\text { (See Table 2) }\end{array}$} & \multicolumn{2}{|c|}{ SG } & \multicolumn{2}{|c|}{ PAC } & \multicolumn{2}{|c|}{ CB $400 \mathrm{~m}$} & \multicolumn{2}{|c|}{ CB $800 \mathrm{~m}$} & \multicolumn{2}{|c|}{ SA $400 \mathrm{~m}$} & \multicolumn{2}{|c|}{ SA $800 \mathrm{~m}$} \\
\hline & $\begin{array}{l}\text { Mean } \\
\text { (SD) }\end{array}$ & $\begin{array}{l}\text { Min } \\
\text { Max }\end{array}$ & $\begin{array}{l}\text { Mean } \\
\text { (SD) }\end{array}$ & $\begin{array}{l}\text { Min } \\
\text { Max }\end{array}$ & $\begin{array}{l}\text { Mean } \\
\text { (SD) }\end{array}$ & $\begin{array}{l}\text { Min } \\
\text { Max }\end{array}$ & $\begin{array}{l}\text { Mean } \\
\text { (SD) }\end{array}$ & $\begin{array}{l}\text { Min } \\
\text { Max }\end{array}$ & $\begin{array}{l}\text { Mean } \\
\text { (SD) }\end{array}$ & $\begin{array}{l}\text { Min } \\
\text { Max }\end{array}$ & $\begin{array}{l}\text { Mean } \\
\text { (SD) }\end{array}$ & \\
\hline Small business & $\begin{array}{c}5.6 \\
(7.89)\end{array}$ & $\begin{array}{c}0.00 \\
85.00\end{array}$ & $\begin{array}{l}28.30 \\
(32.00)\end{array}$ & $\begin{array}{c}0.00 \\
375.00\end{array}$ & $\begin{array}{c}70.60 \\
(118.00)\end{array}$ & $\begin{array}{c}0.00 \\
1060.00\end{array}$ & $\begin{array}{l}209.00 \\
(291.00)\end{array}$ & $\begin{array}{c}1.00 \\
1810.00\end{array}$ & $\begin{array}{l}29.00 \\
(59.70)\end{array}$ & $\begin{array}{c}0.00 \\
808.00\end{array}$ & $\begin{array}{c}86.30 \\
(168.00)\end{array}$ & $\begin{array}{c}0.00 \\
1640.00\end{array}$ \\
\hline $\begin{array}{l}\text { Groceries, } \\
\text { and supermarkets }\end{array}$ & $\begin{array}{c}6.52 \\
(6.50)\end{array}$ & $\begin{array}{c}0.00 \\
65.00\end{array}$ & $\begin{array}{l}21.80 \\
(12.80)\end{array}$ & $\begin{array}{c}0.00 \\
108.00\end{array}$ & $\begin{array}{l}34.60 \\
(29.80)\end{array}$ & $\begin{array}{c}0.00 \\
179.00\end{array}$ & $\begin{array}{l}92.00 \\
(72.80)\end{array}$ & $\begin{array}{c}0.00 \\
411.00\end{array}$ & $\begin{array}{l}14.50 \\
(17.60)\end{array}$ & $\begin{array}{c}0.00 \\
143.00\end{array}$ & $\begin{array}{l}37.20 \\
(43.50)\end{array}$ & $\begin{array}{c}0.00 \\
275.00\end{array}$ \\
\hline Entertainments & $\begin{array}{c}7.66 \\
(9.49)\end{array}$ & $\begin{array}{c}0.00 \\
116.00\end{array}$ & $\begin{array}{l}38.50 \\
(29.40)\end{array}$ & $\begin{array}{c}0.00 \\
398.00\end{array}$ & $\begin{array}{l}70.80 \\
(98.40)\end{array}$ & $\begin{array}{c}0.00 \\
802.00\end{array}$ & $\begin{array}{l}207.00 \\
(238.00)\end{array}$ & $\begin{array}{c}3.00 \\
1530.00\end{array}$ & $\begin{array}{l}28.70 \\
(54.60)\end{array}$ & $\begin{array}{c}0.00 \\
687.00\end{array}$ & $\begin{array}{c}82.60 \\
(143.00)\end{array}$ & $\begin{array}{c}0.00 \\
1140.00\end{array}$ \\
\hline $\begin{array}{l}\text { Government and } \\
\text { institutions }\end{array}$ & $\begin{array}{c}3.88 \\
(4.56)\end{array}$ & $\begin{array}{c}0.00 \\
53.00\end{array}$ & $\begin{array}{l}15.50 \\
(13.40)\end{array}$ & $\begin{array}{c}0.00 \\
134.00\end{array}$ & $\begin{array}{c}29.50 \\
(49.60)\end{array}$ & $\begin{array}{c}0.00 \\
355.00\end{array}$ & $\begin{array}{c}85.80 \\
(123.00)\end{array}$ & $\begin{array}{c}0.00 \\
662.00\end{array}$ & $\begin{array}{l}11.60 \\
(24.40)\end{array}$ & $\begin{array}{c}0.00 \\
281.00\end{array}$ & $\begin{array}{l}34.50 \\
(69.90)\end{array}$ & $\begin{array}{c}0.0 \\
559.00\end{array}$ \\
\hline Education & $\begin{array}{c}7.55 \\
(7.60)\end{array}$ & $\begin{array}{c}0.00 \\
100.00\end{array}$ & $\begin{array}{l}28.10 \\
(18.40)\end{array}$ & $\begin{array}{c}0.00 \\
161.00\end{array}$ & $\begin{array}{c}43.00 \\
(49.50)\end{array}$ & $\begin{array}{c}0.00 \\
398.00\end{array}$ & $\begin{array}{c}125.00 \\
(127.00)\end{array}$ & $\begin{array}{c}1.00 \\
789.00\end{array}$ & $\begin{array}{c}16.80 \\
(27.40)\end{array}$ & $\begin{array}{c}0.00 \\
340.00\end{array}$ & $\begin{array}{l}47.50 \\
(73.70)\end{array}$ & $\begin{array}{c}0.00 \\
597.00\end{array}$ \\
\hline Hotels & $\begin{array}{c}0.4 \\
(3.85)\end{array}$ & $\begin{array}{c}0.00 \\
347.00\end{array}$ & $\begin{array}{c}2.40 \\
(10.50)\end{array}$ & $\begin{array}{c}0.00 \\
638.00\end{array}$ & $\begin{array}{l}12.50 \\
(51.50)\end{array}$ & $\begin{array}{c}0.00 \\
901.00\end{array}$ & $\begin{array}{c}40.10 \\
(131.00)\end{array}$ & $\begin{array}{c}0.00 \\
1260.00\end{array}$ & $\begin{array}{c}5.99 \\
(32.60)\end{array}$ & $\begin{array}{c}0.00 \\
887.00\end{array}$ & $\begin{array}{l}19.00 \\
(81.10)\end{array}$ & $\begin{array}{c}0.00 \\
990.00\end{array}$ \\
\hline Retails & $\begin{array}{c}21.70 \\
(50.00)\end{array}$ & $\begin{array}{c}0.00 \\
597.00\end{array}$ & $\begin{array}{c}93.50 \\
(118.00)\end{array}$ & $\begin{array}{c}0.00 \\
1310.00\end{array}$ & $\begin{array}{l}200.00 \\
(341.00)\end{array}$ & $\begin{array}{c}0.00 \\
2830.00\end{array}$ & $\begin{array}{c}579.00 \\
(825.00)\end{array}$ & $\begin{array}{c}0.00 \\
4770.00\end{array}$ & $\begin{array}{c}84.10 \\
(183.00)\end{array}$ & $\begin{array}{c}0.00 \\
2320.00\end{array}$ & $\begin{array}{c}247.00 \\
(497.00)\end{array}$ & $\begin{array}{c}0.00 \\
3880.00\end{array}$ \\
\hline Companies & $\begin{array}{c}10.60 \\
(52.00)\end{array}$ & $\begin{array}{c}0.00 \\
2000.00\end{array}$ & $\begin{array}{c}149.00 \\
(471.00)\end{array}$ & $\begin{array}{c}0.00 \\
5170.00\end{array}$ & $\begin{array}{c}353.00 \\
(753.00)\end{array}$ & $\begin{array}{c}0.00 \\
6580.00\end{array}$ & $\begin{array}{c}1210.00 \\
(1930.00)\end{array}$ & $\begin{array}{c}0.00 \\
11200.00\end{array}$ & $\begin{array}{c}130.00 \\
(360.00)\end{array}$ & $\begin{array}{c}0.00 \\
5380.00\end{array}$ & $\begin{array}{c}443.00 \\
(1010.00)\end{array}$ & $\begin{array}{c}0.00 \\
0400.00\end{array}$ \\
\hline Health care services & $\begin{array}{c}7.13 \\
(8.03)\end{array}$ & $\begin{array}{c}0.00 \\
149.00\end{array}$ & $\begin{array}{c}30.60 \\
(29.20)\end{array}$ & $\begin{array}{c}0.00 \\
522.00\end{array}$ & $\begin{array}{c}63.60 \\
(112.00)\end{array}$ & $\begin{array}{c}0.00 \\
1200.00\end{array}$ & $\begin{array}{c}181.00 \\
(272.00)\end{array}$ & $\begin{array}{c}0.00 \\
1690.00\end{array}$ & $\begin{array}{l}27.70 \\
(69.40)\end{array}$ & $\begin{array}{c}0.00 \\
1200.00\end{array}$ & $\begin{array}{c}79.70 \\
(175.00)\end{array}$ & $\begin{array}{c}0.00 \\
1540.00\end{array}$ \\
\hline Financial services & $\begin{array}{c}2.99 \\
(5.03)\end{array}$ & $\begin{array}{l}0.00 \\
61.00\end{array}$ & $\begin{array}{l}13.20 \\
(16.50)\end{array}$ & $\begin{array}{c}0.00 \\
242.00\end{array}$ & $\begin{array}{l}31.10 \\
(58.70)\end{array}$ & $\begin{array}{c}0.00 \\
730.00\end{array}$ & $\begin{array}{c}89.20 \\
(138.00)\end{array}$ & $\begin{array}{c}0.00 \\
1140.00\end{array}$ & $\begin{array}{l}12.80 \\
(29.80)\end{array}$ & $\begin{array}{c}0.00 \\
633.00\end{array}$ & $\begin{array}{l}37.60 \\
(81.30)\end{array}$ & $\begin{array}{c}0.00 \\
1080.00\end{array}$ \\
\hline Restaurants & $\begin{array}{c}19.10 \\
(25.30)\end{array}$ & $\begin{array}{c}0.00 \\
223.00\end{array}$ & $\begin{array}{c}83.30 \\
(69.80)\end{array}$ & $\begin{array}{c}0.00 \\
650.00\end{array}$ & $\begin{array}{c}162.00 \\
(213.00)\end{array}$ & $\begin{array}{c}0.00 \\
1600.00\end{array}$ & $\begin{array}{c}451.00 \\
(507.00)\end{array}$ & $\begin{array}{c}0.00 \\
2850.00\end{array}$ & $\begin{array}{c}68.10 \\
(114.00)\end{array}$ & $\begin{array}{c}0.00 \\
1250.00\end{array}$ & $\begin{array}{c}187.00 \\
(298.00)\end{array}$ & $\begin{array}{c}0.00 \\
2100.00\end{array}$ \\
\hline $\begin{array}{l}\text { Distance to transit } \\
\text { Density of transit } \\
\text { services } \\
\text { Quantity of transit } \\
\text { services }\end{array}$ & $\begin{array}{c}0.00006 \\
(0.00008) \\
4.50 \\
(5.42)\end{array}$ & $\begin{array}{c}0.00 \\
0.00113 \\
0.00 \\
28.00\end{array}$ & $\begin{array}{c}0.00004 \\
(0.00004) \\
20.70 \\
(14.10)\end{array}$ & $\begin{array}{c}0.00 \\
0.00036 \\
0.00 \\
104.00\end{array}$ & $\begin{array}{c}0.00005 \\
(0.00003) \\
23.70 \\
(13.30)\end{array}$ & $\begin{array}{c}0.00 \\
0.00017 \\
0.00 \\
84.00\end{array}$ & $\begin{array}{c}0.00003 \\
(0.00002) \\
69.40 \\
(39.20)\end{array}$ & $\begin{array}{c}0.00 \\
0.00010 \\
3.00 \\
208.00\end{array}$ & $\begin{array}{c}0.00006 \\
(0.00004) \\
8.90 \\
(8.00)\end{array}$ & $\begin{array}{c}0.00 \\
0.00037 \\
0.00 \\
61.00\end{array}$ & $\begin{array}{c}0.00005 \\
(0.00003) \\
24.80 \\
(22.10)\end{array}$ & $\begin{array}{c}0.00 \\
0.00025 \\
0.00 \\
158.00\end{array}$ \\
\hline
\end{tabular}

SG, Street-block group; PAC, primary adjacency community; CB, circular buffer; SA, service area buffer. 
to the SG level (3.77). CB_800m indicated the lowest building density (1.85) which was only half of the counterpart using the SA buffer. Judged by the diversity measures, the destination mixture was the greatest at the SG level $(0.48)$, followed by SA $400 \mathrm{~m}$ (0.42), PAC (0.36), SA_800m (0.36) and CB_400m (0.34). CB_800m $(0.28)$ had the smallest diversity in mixture of the destinations/services.

Judged by the design measures, the median block lengths were not consistent with the average block lengths. For example, the median length was $80.2 \mathrm{~m}$ compared to a mean of $118.0 \mathrm{~m}$ at the SG level. SA_800m had the smallest median length $(51.0 \mathrm{~m})$ among all spatial scales, while SG had the longest block length both with respect to the median and average measures. SA buffers had greater density of street intersections than the $\mathrm{CB}$ counterparts (e.g., 0.00145 for SA $400 \mathrm{~m}$ versus 0.00038 for $\mathrm{CB} 400 \mathrm{~m}$ ). Intriguingly, SG having the lowest connectivity measured using median bock length, that is having the longest block length (80.20), is reversed to have the highest connectivity measured using link-node ratio (0.85).

Judged by the destination accessibility measures, SG had the smallest quantity of destinations in most of the destination categories, while CB_800m held the largest quantity of destinations and showed striking differences between scales in categories of small business (5.62 versus 209.00), retails (21.70 versus 579.00), companies (10.60 versus 1210.00), and restaurants (19.10 versus 451.00). The quantities of destinations in SA_400m was less than half the quantity of destinations in $\mathrm{CB} \_400 \mathrm{~m}$ counterparts (e.g., educational (16.80 versus 43.00), entertainments (28.70 versus $70.80)$, and restaurants (68.10 versus 162.00$)$ ). The differences in destination quantities were offset or even reversed compared to the destination density measures. For example, retail density between $\mathrm{SG}$ and $\mathrm{CB} 8800 \mathrm{~m}$ was reversed compared to the absolute measure: quantity was 21.70 versus 579.00 , while the density was 0.00053 per $\mathrm{m}^{2}$ versus 0.00029 per $\mathrm{m}^{2}$. Generally, SG had the largest density of destinations of all the categories (except for the company category).

Judged by the distance to transit measures, SG and SA $400 \mathrm{~m}$ had the largest density of transit (0.00006), while CB_800m had the smallest (0.00003). The quantity measures preserve the density ranking across spatial scales, with $\mathrm{CB}$ _800m having the largest amount of transit, 68.4 stops being within accessible distance on average compared to 4.5 at the SG level.

\section{Mapping built environment measurement uncertainty}

We used a set of maps to show the diversity of destination mix contra density and quantity of retail as examples of variability in measures across spatial scales when measuring the high-density BE of Hong Kong (Figure 3). Destination mixture was evenly distributed across the territory using SG. There was no clear difference, on this measure, between new towns in the New Territories and the city centre on Hong Kong Island. On the contrary, the distribution of service mix was much more uneven using SA_400m. The areas with relative low service mix were in the northern part of Hong Kong Island (from Sheung Wan to Causeway Bay) and the linear area from Tsim Sha Tsui to Mong Kok (around Nathan Road) in Kowloon. These were both among the most densely populated areas in Hong Kong.

Quantity measures of the retail service were quite different comparing SG and SA_400m. Most of the places which had less than 20 retail stores in the block measure had more than 100 in the $400 \mathrm{~m}$ network buffer measure. The densest places measured in the retails quantity were in similar areas that had the relative lower destination mix in the northern part of Hong Kong island and around Nathan Road. The variations in retail density between the two spatial scales were much less than with the quantity measure, except for a few places such as the eastern part of Hong Kong island and the linear area from Hung Ham to To Kwa Wan in Kowloon. Similar degrees and patterns of uncertainty over spatial scale were found in most of the other destination categories referred in Tables 4-6.

\section{Statistical analyses of variability/uncertainty}

Spearman's rank correlations for BE measures across scales are shown in Table 7. Judged by the density measures, block-level building density correlated weakly with PAC, CB_400m, and SA_800m $(\rho>0.30$ and $\rho<=0.50)$. It did not correlate with CB_800m $(\rho=0.27)$. PAC had a weak correlation with SA_400m, a moderate correlation with SA_800m $(\rho=0.55)$ and a strong correlation with $\mathrm{CB} \_400 \mathrm{~m}$ and $\mathrm{CB} \_800 \mathrm{~m}(\rho>0.70)$. CB_400m had a strong correlation with $\mathrm{CB}_{-} 800 \mathrm{~m}$, SA $400 \mathrm{~m}$, and SB800 $(\rho>0.70)$. CB_800m had a weak correlation with SA_400m and a moderate correlation with SA_800m $(\rho=0.61)$. A strong correlation was found with SA_400m and SA_800m $(\rho=0.83)$.

Judged by the diversity measures, the mix of services had a weak correlation with SG and SA_400m, SG and SA_800m, PAC and CB_800m, CB_800m and SA_400m $(\rho=0.32 \sim 0.48)$. There was no correlation between SG and CB_800m $(\rho=0.17)$. A wellpreserved correlation was shown between SA_400m and $\mathrm{CB} 800 \mathrm{~m}, \mathrm{CB} 400 \mathrm{~m}$ and $\mathrm{CB} 800 \mathrm{~m}$, and $\mathrm{CB} 400 \mathrm{~m}$ and SA_800m $(\rho=0.72 \sim 0.80)$.

Judged by the design measures, median block length was less consistent across spatial scales than average block length except when comparing SG and PAC ( $\rho=0.45$ versus 0.40$)$, and SA $400 \mathrm{~m}$ and SA_800m $(\rho=0.86$ versus 0.84$)$. The average block length exhibits weak to moderate correlations among the six spatial scales ( $\rho=0.34 \sim 0.67)$, with the exception of a non-correlated relationship between SG and CB_800m $(\rho=0.23)$ and a high correlation between SA_400m and SA_800m $(\rho=0.84)$. Link-node ratio showed extreme variability across scales. Most rankings were not preserved $(\rho=-0.09 \sim 0.25)$, except weak correlations between CB $400 \mathrm{~m}$ and SA $800 \mathrm{~m}(\rho=0.32)$ and SA $400 \mathrm{~m}$ and SA $800 \mathrm{~m}$ $(\rho=0.48)$ and a moderate correlation between SG and PAC $(\rho=0.57)$.

Judged by the destination accessibility measures, rank correlations of service destination density measures were preserved better than service destination quantity measures in general. For example, density measures of accessible entertainments shown moderate to high correlation between scales $(\rho=0.43 \sim 0.85)$, while quantity measures in SG were not correlated with $\mathrm{CB}_{-} 400 \mathrm{~m}(\rho=0.11)$, CB_800m $(\rho=-0.03)$, SA_400m $(0.18)$, or SA_800m $(0.11)$.

Judged by the distance to transit measures, we observed weak correlations for density measures of accessible transit comparing SG and the other scales $(\rho=0.25 \sim 0.37)$. However, the SG measures of accessible transit counts were not preserved with the other scales $(\rho=(-0.14) \sim(-0.02))$ except with PAC $(\rho=0.72)$. Similarly, PAC measures of quantity were not correlated with $C B \_400 \mathrm{~m}$, CB_800m, SA_400m, and SA_800m $(\rho=-0.06 \sim 0.07)$.

\section{Discussion}

This paper deals with our understanding of uncertainty, a long- 

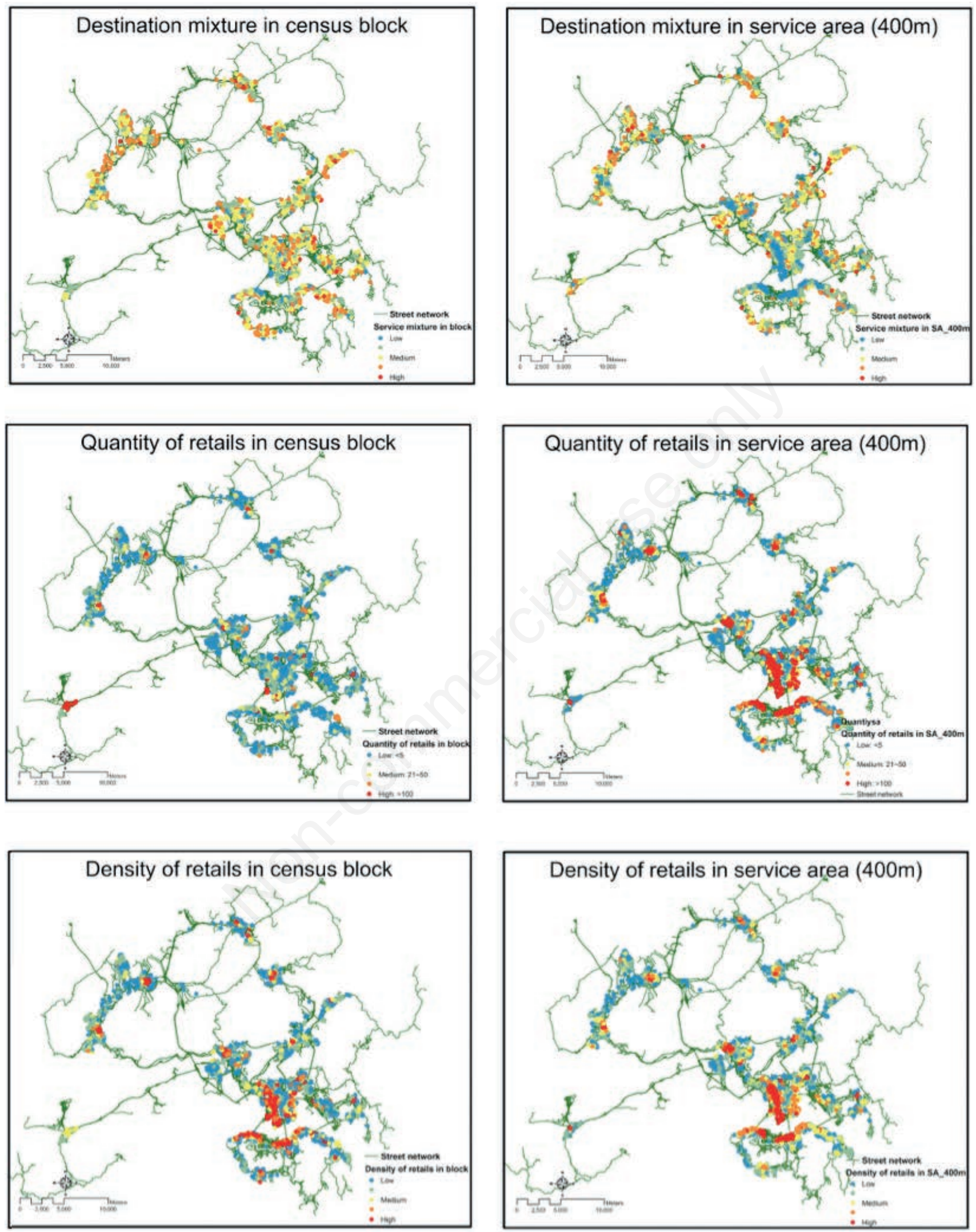

Figure 3. Examples for the uncertainty of construction methods and spatial scales in measuring the high density built environment of Hong Kong. 
Table 7. Spearman's correlations among built environment characteristics in scales.

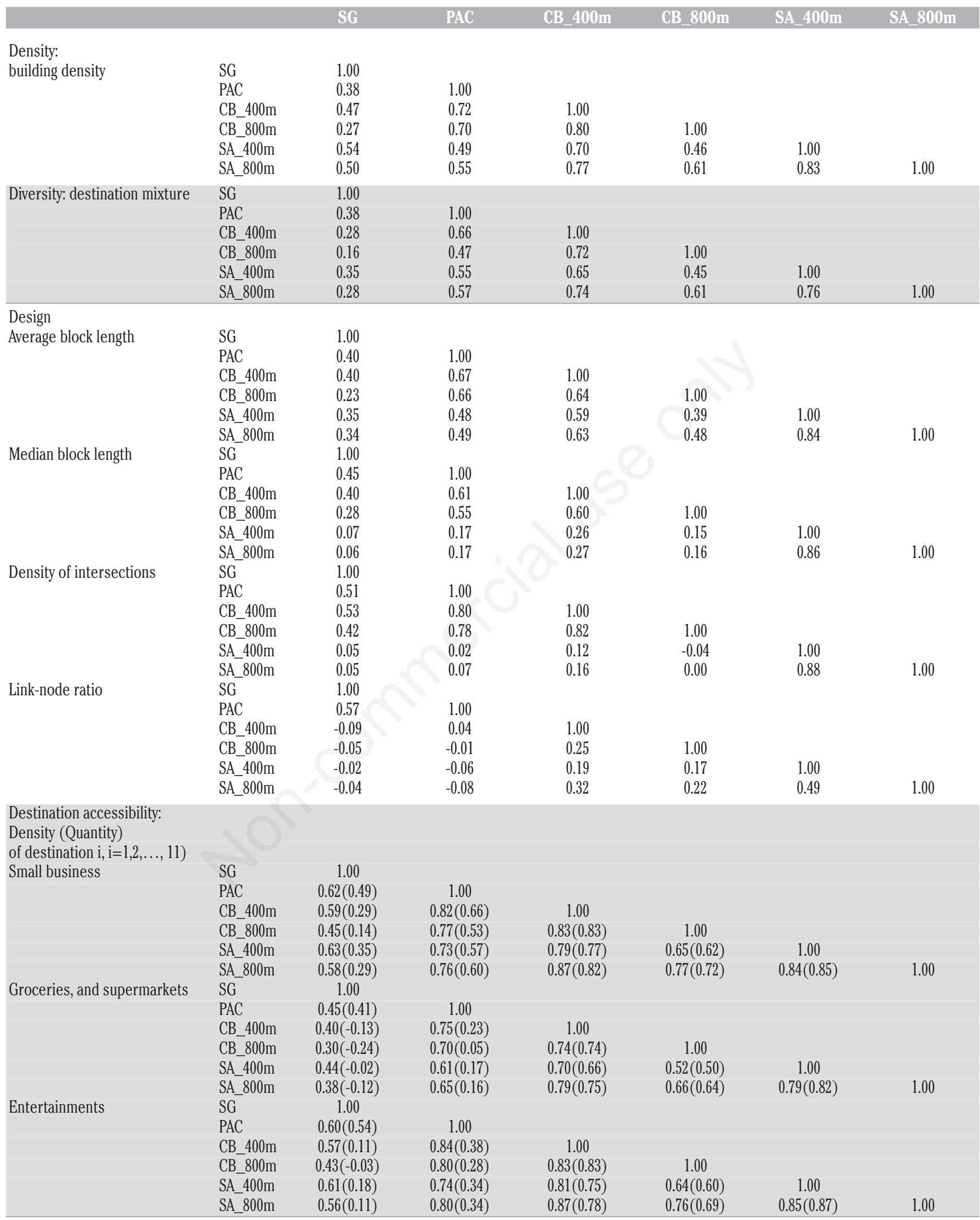

To be continued on the next page. 


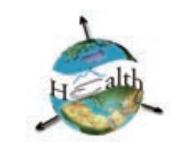

standing but less studied issue in urban health, with a particular focus in high-density and heterogonous urban situations. We examined the uncertainty in measuring high-density BE, as typified by Hong Kong, with a view to understand the variability of BE measures across different design methods and spatial units. Our results reveal how variability affects the sensitivity of BE measures in health studies.

We constructed $30 \mathrm{BE}$ indicators of land use patterns, transport

Table 7. Continued from previous page.

\begin{tabular}{|c|c|c|c|c|c|c|c|}
\hline & & SG & PAC & CB_400m & CB_800m & SA_ $400 \mathrm{~m}$ & SA_800m \\
\hline \multirow[t]{6}{*}{ Government and institutions } & SG & 1.00 & & & & & \\
\hline & $\mathrm{PAC}$ & $0.36(0.52)$ & 1.00 & & & & \\
\hline & CB_400m & $0.41(0.12)$ & $0.75(0.44)$ & 1.00 & & & \\
\hline & CB_800m & $0.26(-0.06)$ & $0.73(0.29)$ & $0.79(0.79)$ & 1.00 & & \\
\hline & SA_400m & $0.46(0.16)$ & $0.63(0.37)$ & $0.75(0.72)$ & $0.59(0.57)$ & 1.00 & \\
\hline & SA_800m & $0.40(0.04)$ & $0.69(0.33)$ & $0.84(0.77)$ & $0.72(0.68)$ & $0.82(0.81)$ & 1.00 \\
\hline \multirow[t]{6}{*}{ Educational } & SG & 1.00 & & & & & \\
\hline & $\mathrm{PAC}$ & $0.25(0.52)$ & 1.00 & & & & \\
\hline & CB_400m & $0.26(-0.16)$ & $0.75(0.21)$ & 1.00 & & & \\
\hline & CB_800m & $0.13(-0.32)$ & $0.74(0.08)$ & $0.78(0.78)$ & 1.00 & & \\
\hline & SA_400m & $0.35(-0.05)$ & $0.60(0.17)$ & $0.70(0.64)$ & $0.53(0.51)$ & 1.00 & \\
\hline & SA_800m & $0.25(-0.18)$ & $0.65(0.12)$ & $0.80(0.71)$ & $0.68(0.64)$ & $0.79(0.83)$ & 1.00 \\
\hline \multirow[t]{6}{*}{ Hotels } & $\mathrm{SG}$ & 1.00 & & & & & \\
\hline & PAC & $0.51(0.54)$ & 1.00 & & & & \\
\hline & CB_400m & $0.40(0.38)$ & $0.73(0.70)$ & 1.00 & & & \\
\hline & CB_800m & $0.29(0.28)$ & $0.59(0.56)$ & $0.77(0.77)$ & 1.00 & & \\
\hline & SA_400m & $0.46(0.44)$ & $0.71(0.67)$ & $0.79(0.79)$ & $0.64(0.64)$ & 1.00 & \\
\hline & SA_800m & $0.38(0.36)$ & $0.69(0.64)$ & $0.85(0.84)$ & $0.74(0.74)$ & $0.83(0.83)$ & 1.00 \\
\hline \multirow[t]{6}{*}{ Retails } & $\mathrm{SG}$ & 1.00 & & & & & \\
\hline & $\mathrm{PAC}$ & $0.61(0.50)$ & 1.00 & & & & \\
\hline & CB_400m & $0.56(0.32)$ & $0.77(0.61)$ & 1.00 & & & \\
\hline & CB_800m & $0.43(0.18)$ & $0.67(0.44)$ & $0.72(0.72)$ & 1.00 & & \\
\hline & $\mathrm{SA} \quad 400 \mathrm{~m}$ & $0.60(0.35)$ & $0.68(0.48)$ & $0.73(0.71)$ & $0.54(0.53)$ & 1.00 & \\
\hline & SA_800m & $0.55(0.30)$ & $0.74(0.55)$ & $0.82(0.79)$ & $0.67(0.66)$ & $0.82(0.84)$ & 1.00 \\
\hline \multirow[t]{6}{*}{ Companies } & $\mathrm{SG}$ & 1.00 & & & & & \\
\hline & $\mathrm{PAC}$ & $0.51(0.47)$ & 1.00 & & & & \\
\hline & CB_400m & $0.44(0.33)$ & $0.77(0.67)$ & 1.00 & & & \\
\hline & CB_800m & $0.29(0.16)$ & $0.68(0.53)$ & $0.80(0.80)$ & 1.00 & & \\
\hline & SA_400m & $0.54(0.42)$ & $0.69(0.56)$ & $0.72(0.70)$ & $0.57(0.55)$ & 1.00 & \\
\hline & SA_800m & $0.47(0.35)$ & $0.72(0.59)$ & $0.81(0.78)$ & $0.70(0.67)$ & $0.82(0.83)$ & 1.00 \\
\hline \multirow[t]{6}{*}{ Health care services } & SG & 1.00 & & & & & \\
\hline & $\mathrm{PAC}$ & $0.59(0.54)$ & 1.00 & & & & \\
\hline & CB_400m & $0.49(0.10)$ & $0.78(0.42)$ & 1.00 & & & \\
\hline & CB_800m & $0.37(-0.04)$ & $0.71(0.27)$ & $0.77(0.77)$ & 1.00 & & \\
\hline & SA_400m & $0.55(0.17)$ & $0.68(0.34)$ & $0.74(0.70)$ & $0.57(0.55)$ & 1.00 & \\
\hline & SA_800m & $0.49(0.10)$ & $0.72(0.37)$ & $0.84(0.78)$ & $0.70(0.67)$ & $0.81(0.84)$ & 1.00 \\
\hline \multirow[t]{6}{*}{ Financial services } & $\mathrm{SG}^{-}$ & 1.00 & & & & & \\
\hline & PAC & $0.40(0.46)$ & 1.00 & & & & \\
\hline & CB_400m & $0.28(0.15)$ & $0.70(0.52)$ & 1.00 & & & \\
\hline & CB_800m & $0.16(-0.01)$ & $0.64(0.33)$ & $0.72(0.72)$ & 1.00 & & \\
\hline & SA_400m & $0.37(0.23)$ & $0.64(0.46)$ & $0.71(0.70)$ & $0.52(0.52)$ & 1.00 & \\
\hline & SA_800m & $0.29(0.14)$ & $0.66(0.45)$ & $0.81(0.79)$ & $0.66(0.66)$ & $0.78(0.80)$ & 1.00 \\
\hline \multirow[t]{6}{*}{ Restaurants } & SG & 1.00 & & & & & \\
\hline & PAC & $0.60(0.46)$ & 1.00 & & & & \\
\hline & CB_400m & $0.58(0.16)$ & $0.79(0.51)$ & 1.00 & & & \\
\hline & CB_800m & $0.47(0.03)$ & $0.74(0.36)$ & $0.78(0.78)$ & 1.00 & & \\
\hline & SA_400m & $0.63(0.23)$ & $0.72(0.43)$ & $0.78(0.75)$ & $0.61(0.59)$ & 1.00 & \\
\hline & SA_800m & $0.58(0.16)$ & $0.74(0.46)$ & $0.84(0.80)$ & $0.72(0.70)$ & $0.84(0.86)$ & 1.00 \\
\hline \multirow{6}{*}{$\begin{array}{l}\text { Distance to transit: density } \\
\text { (quantity) of transit services }\end{array}$} & SG & 1.00 & & & & & \\
\hline & PAC & $0.37(0.72)$ & 1.00 & & & & \\
\hline & CB_400m & $0.35(-0.03)$ & $0.75(0.07)$ & 1.00 & & & \\
\hline & CB_800m & $0.25(-0.14)$ & $0.75(0.00)$ & $0.81(0.81)$ & 1.00 & & \\
\hline & SA_400m & $0.35(-0.02)$ & $0.58(0.02)$ & $0.70(0.61)$ & $0.55(0.48)$ & 1.00 & \\
\hline & SA_800m & $0.31(-0.13)$ & $0.65(-0.06)$ & $0.81(0.67)$ & $0.72(0.62)$ & $0.76(0.82)$ & 1.00 \\
\hline
\end{tabular}

SG, Street-block group; PAC, Primary adjacency community; CB_400m, Circular buffer in 400m; CB_800m, Circular buffer in 800m; SA_400m, Network area buffer in 400m; SA_800m, Network area buffer in 800m. 
and urban design around 5,732 geocoded residential addresses of Hong Kong FAMILY Cohort members. Each indicator was calculated at six spatial scales, commonly applied in health-related behavioural studies, adopting the 5-D BE framework to increase comparability with international studies. These urban morphology metrics were constructed based on a complete database; specifically, we measured destination/service diversity from a complete suite of points of interest in detailed categories. It is different from pervious diversity measures using mixture indices constructed at the parcel level (e.g. residential, commercial, or industrial levels) with abstract land use categories (Frank et al., 2005). The abstract land use at the parcel level is unable to capture the variations in highdensity environments with intense mixed-use development, which may lead to invalid findings regarding the linkages of BE-health outcomes (Lu et al., 2016). We posed a detailed taxonomy of the classification of services for destination accessibility measures, which can serve as a protocol for future studies.

We found a high variability in construction methods of $\mathrm{BE}$ indicators. For example, significant differences in the urban design dimension appear between block length (median and average) and street connectivity (density of intersections and link-node ratios), and between quantity measures of destination accessibility and their counterparts in density measures. Median block length and link-node ratio showed less preserved correlations in ranking than other measures of the design dimension, the median being more sensitive than the average for measuring variability of the block size. Measurement rankings can even be reversed in different design methods of indicators. For example, the lowest connectivity measured by the median bock length at the SG level was shown to have the highest connectivity in link-node ratio on the contrary, which may lead to extreme inconsistence when applying them separately in regression models. Quantity measures of service destination accessibility were found to be more sensitive than the service density measures. These uncertainties are somewhat in line with the findings in low-density cities (Mitra and Buliung, 2012; Clark and Scott, 2014; Strominger et al., 2016), but the uncertainty level is more pronounced in the high-density environment.

We found considerable uncertainties in measuring a high-density BE across spatial scales. Most of measures using the smallest census tract of Hong Kong, the SG scale, were quite inconsistent with other scales, indicating minimal correlation. The census tract scale may therefore be inappropriate for capturing variability of urban morphology in high-density building areas, although it has the advantage of correlating BE measures with census variables. This calls attention to health research using census tracts in similar high-density contexts. Likewise, we found none or weak correlations when comparing indicator rankings at the CB_800m levels with other scales. The poorly preserved correlations between $\mathrm{CB}$ and others may partially originate from the constraint layout of the urban area in Hong Kong, where developed land is squeezed between mountains and the sea. CB used in this morphological context is likely to include a large proportion of uninhabited areas of mountains or water body. Similarly, larger zonal PAC measures perform poorly because they tend to involve inaccessible spaces due to ownership or institutional, configurational, slope and other reasons. Models of BE-health associations using these scales could, therefore, fail to infer valid linkages between BE and health impact. On the contrary, a network buffer, which is less contaminated by inaccessible spaces, becomes a more appropriate unit for analysis.

\section{Implications}

The uncertainty demands reflection with regard to design methods and spatial scales when constructing BE indicators for health studies. Qualitative interviews of subjects would be required to gain a better understanding of activity space, thus informing selection of BE metrics to better reflect a subject's geographic context. Taking the quantity and density of accessible destinations as an example, destination counts are better at reflecting a location's vitality than destination density. However, subjects may perceive no difference in utility between neighbourhood environments with 10 20 restaurants and another with $20 \sim 30$. It therefore matters that the two types of measures have significantly different rankings when measured at different scales and with different shapes. Density measures will be biased as an indicator of place viability, due to intrinsic lack of correlation with the more important destination count morphology.

Researchers need to use a scale that is appropriate to the behaviours investigated. When constructing a BE measure, subjects may be asked, for example, to actively draw what they consider their behavioural space to be, or passively reveal the ideal spatial scales for some particular choice set (e.g., active trajectories by GPS) (Tribby et al., 2017). An extension of our study would be to repeat the systematic comparison of BE measures across scales within the context of a BE-health associational study. In this way, the impact of using certain measures rather than others could be assessed in terms of correlation coefficients, not just in terms of the descriptive performance of the measures compared against each other.

\section{Limitation and strengths}

This study has several limitations, e.g., we did not consider the weights of different destinations/services in measuring the diversity of land use. Weighting would be needed when linking these indicators to health outcomes. For example, retail destinations may be more important for walking studies than small businesses, which need to be differentiated from companies. We used a street network rather than a pedestrian network for the urban design measures and there may be behavioural differences between those two networks (Sun et al., 2015). The study was conducted in a high-density urban environment, so generalization of the finding should be done with caution. However, the methodology can be reliably adapted to other high-density cities and elsewhere, with additional considerations. Finally, we only discussed static BE exposure measures based on residential addresses, while dynamic measures of exposure may be required to connect 'activity spaces' with exposures in different environments. Understanding the spatial and temporal variations of exposure is critical when dynamic exposures are required (Kwan, 2013; Burgoine et al., 2014; Tenailleau et al., 2015).

To the best of our knowledge, this is the first study to systematically profile variability of $\mathrm{BE}$ measures across scale and shape of measurement buffers in a high-density city. This is a notable strength as this approach is fundamental for research and practice for promoting healthy high-density cities. We can further explore the linkage of $\mathrm{BE}$ and health behaviours and disentangle the impact of $\mathrm{BE}$ on health through rigorous and solid measures, which represents evidence of the need for effective BE intervention in high- density cities for urban planners, policy makers and public health practitioners. We are working on building an open data web platform to support scholars who are interested in studies of BE-health associations in Hong Kong. The methodologies, 
including data collection protocols, measures, and modelling provide a benchmark for high density BE-health studies in Hong Kong and other high-density cities around the world.

\section{Conclusions}

We measured and classified attributes of the high-density BE based on a comprehensive urban dataset which is essential for testing health-related research hypotheses. Our findings suggest complete data, appropriate design methods and suitable spatial scales of measures are crucial in high density BE related health studies. Some indictors were found to be more robust than others. When high-density urban space is linearly constrained by uninhabited areas, as in Hong Kong, network buffers likely to retain more meaningful activity space than circular buffers while circular buffers and block-based geography, with the latter's attraction of linked census data, may be more acceptable surrogates in homogenous lower density cities. This is the first study to systematically examine $\mathrm{BE}$ in a high-density city, which can be framework of measuring BE for healthy studies in other similar contexts.

\section{References}

Adams MA, Frank LD, Schipperijn J, Smith G, Chapman J, Christiansen LB, Coffee N, Salvo D, du Toit L, Dygrýn J, Hino AA, Lai P, Mavoa S, Pinzón J, Van de Weghe N, Cerin E, Davey R, Macfarlane D, Owen N, Sallis JF, 2014. International variation in neighborhood walkability, transit, and recreation environments using geographic information systems: the IPEN adult study. Int J Health Geogr 13:43.

Barton H, Grant M, 2013. Urban planning for healthy cities a review of the progress of the european healthy cities programme. J Urban Heal 90:129-41.

Berrigan D, Pickle LW, Dill J, 2010. Associations between street connectivity and active transportation. Int J Health Geogr 9:20.

Brownson RC, Hoehner CM, Day K, Forsyth A, Sallis JF, 2009. Measuring the built environment for physical activity: state of the science. Am J Prev Med 36:S99-123.e12.

Burgoine T, Forouhi NG, Griffin SJ, Wareham NJ, Monsivais P, 2014. Associations between exposure to takeaway food outlets, takeaway food consumption, and body weight in Cambridgeshire, UK: population based, cross sectional study. Bmj 348:g1464.

Cerin E, Chan K, Macfarlane DJ, Lee K, Lai P, 2011. Objective assessment of walking environments in ultra-dense cities: development and reliability of the environment in Asia Scan Tool-Hong Kong version (EAST-HK). Health Place 17:93745.

Cerin E, Lee K, Barnett A, Sit CH, Cheung M, Chan W, Johnston JM, 2013. Walking for transportation in Hong Kong Chinese urban elders: a cross-sectional study on what destinations matter and when. Int J Behav Nutr Phys Act 10:78.

Clark A, Scott D, 2014. Understanding the impact of the modifiable areal unit problem on the relationship between active travel and the built environment. Urban Stud 51:284-99.

Ewing R, Cervero R, 2010. Travel and the built environment: a meta-analysis. J Am Plan Assoc 76:265-94.

Ewing R, Tian G, Goates J, Zhang M, Greenwald MJ, Joyce A,
Kircher J, Greene W, 2015. Varying influences of the built environment on household travel in 15 diverse regions of the United States. Urban Stud 52:2330-48.

Forsyth A, Schmitz KH, Oakes M, Zimmerman J, Koepp J, 2006. Standards for Environmental Measurement Using GIS: Toward a Protocol for Protocols. J Phys Act Heal 3:241-57.

Frank LD, Schmid TL, Sallis JF, Chapman J, Saelens BE, 2005. Linking objectively measured physical activity with objectively measured urban form: findings from SMARTRAQ. Am J Prev Med 28:117-25.

Handy S, 2005. Critical assessment of the literature on the relationships among transportation, land use, and physical activity. TRB Spec Rep 282:102.

Handy SL, Boarnet MG, Ewing R, Killingsworth RE, 2002. How the built environment affects physical activity: views from urban planning. Am J Prev Med 23:64-73.

Kwan M-P, 2013. Beyond space (as we knew it): toward temporally integrated geographies of segregation, health, and accessibility. Ann Assoc Am Geogr 103:1078-86.

Leal C, Chaix B, 2011. The influence of geographic life environments on cardiometabolic risk factors: A systematic review, a methodological assessment and a research agenda. Obes Rev 12:217-30.

Lee I-M, Shiroma EJ, Lobelo F, Puska P, Blair SN, Katzmarzyk PT, 2012. Effect of physical inactivity on major non-communicable diseases worldwide: an analysis of burden of disease and life expectancy. Lancet 380:219-29.

Leslie E, Coffee N, Frank L, Owen N, Bauman A, Hugo G, 2007. Walkability of local communities: using geographic information systems to objectively assess relevant environmental attributes. Health Place 13:111-22.

Leung GM, Ni MY, Wong PT, Lee PH, Chan BH, Stewart SM, Schooling CM, Johnston JM, Lam WW, Chan SS, McDowell I, Pang H, Fielding R, Lam TH, Pang H, Fielding R, 2015. Cohort Profile: FAMILY Cohort. Int J Epidemiol 46:1-13.

Longley PA, Goodchild MF, Maguire DJ, 2015. Geographic Information Science and Systems. 4th ed. New Jersey, US: Wiley.

Low CT, Lai PC, Li HD, Ho WK, Wong P, Chen S, Wong WC, 2016. Neighbourhood effects on body constitution- a case study of Hong Kong. Soc Sci Med 158:61-74.

Lu Y, Xiao Y, Ye Y, 2016. Urban density, diversity and design: Is more always better for walking? A study from Hong Kong. Prev Med (Baltim) YPMED-0476:1-5.

Mitra R, Buliung RN, 2012. Built environment correlates of active school transportation: neighborhood and the modifiable areal unit problem. J Transp Geogr 20:51-61.

Oliver LN, Schuurman N, Hall AW. 2007. Comparing circular and network buffers to examine the influence of land use on walking for leisure and errands. Int J Health Geogr 6:41.

Rydin Y, Bleahu A, Davies M, Dávila JD, Friel S, De Grandis G, Groce N, Hallal PC, Hamilton I, Howden-Chapman P, Lai KM, Lim CJ, Martins J, Osrin D, Ridley I, Scott I, Taylor M, Wilkinson P, Wilson J, 2012. Shaping cities for health: complexity and the planning of urban environments in the 21 st century. Lancet 379:2079-108.

Sallis JF, Cerin E, Conway TL, Adams MA, Frank LD, Pratt M, Salvo D, Schipperijn J, Smith G, Cain KL, Davey R, Kerr J, Lai P-C, Mitáš J, Reis R, Sarmiento OL, Schofield G, Troelsen J, Van Dyck D, De Bourdeaudhuij I, Owen N, 2016. Physical activity in relation to urban environments in 14 cities world- 
wide: a cross-sectional study. Lancet 387:2207-17.

Shelton B, Karakiewicz J, Kvan T, 2011. The making of hong kong: from vertical to volumetric. London: Routledge.

Strominger J, Anthopolos R, Miranda ML, 2016. Implications of construction method and spatial scale on measures of the built environment. Int J Health Geogr 15:15.

Sun G, Haining R, Lin H, Oreskovic NM, He J, 2015. Comparing the perception with the reality of walking in a hilly environment: an accessibility method applied to a University campus in Hong Kong. Geospat Health 10:340.

Sun G, Webster C, Chiaradia A, 2017. Objective assessment of sta- tion approach routes: Development and reliability of an audit for walking environments around metro stations in China. J Transp Heal 4:191-207.

Tenailleau QM, Bernard N, Pujol S, Houot H, Joly D, Mauny F, 2015. Assessing residential exposure to urban noise using environmental models: does the size of the local living neighborhood matter? J Expo Sci Environ Epidemiol 25:89-96.

Tribby CP, Miller HJ, Brown BB, Smith KR, Werner CM, 2017. Geographic regions for assessing built environmental correlates with walking trips: A comparison using different metrics and model designs. Health Place 45:1-9. 